\title{
Optimization of the Spectrum and Energy Efficiency using Coverage Sensitivity Criterion
}

\author{
K. A. Dotche and Willie K. Ofosu
}

\begin{abstract}
The paper investigated the optimization of both the area spectral efficiency (SE) and the energy efficiency (EE) by considering a typical Orthogonal Frequency Division Multiplexing Access (OFDMA) downlink network. It introduced a novelty constraint, the coverage sensitivity criterion in solving the optimization problem. That constraint aimed at deleting empty transmission block where no cell load was detected. Simulation in Mathlab software was used to solve the problem, and generate the data.
\end{abstract}

Keywords - Theoretical formulation, multi-objective problem, coverage sensitivity, power minimization, spectrum efficiency

\section{Introduction}

The mobile cellular systems [1] operate in a limited spectrum and time slot [2], [3] with high management strategies of the area spectral efficiency (SE), and the energy efficiency (EE). On one hand, the area spectral efficiency could be optimized by the advanced physical layer techniques such as Frequency Division Multiple Access (FDMA), Time Division Multiple Access (TDMA), Code Division Multiple Access (CDMA), Orthogonal Frequency Division Multiple Access (OFDMA), Single Carrier (SC)-OFDMA techniques, Spatial Division Multiple Access (SDMA), [3], [4] etc these multiple access techniques aim at improving the spectral efficiency of the baseband signal at expense of the energy efficiency. An optimal spectral efficiency calls for a higher order modulation scheme [5], which in turn results in a higher power demand. On the other hand, improving the energy efficiency may depend on the Medium Access Control (MAC) protocols [6][7]. In [8], the survey has covered energy efficiency in wireless networks. It has pointed out that the network coding technology has a high potentiality for energy saving. The emphasis on the heterogeneous architecture of multi-cells is developed in [9], meanwhile the cross layers optimization has been suggested in [10]. It has been proven in [11] that the Long Term Evolution network (LTE) power management system relies on the discontinuity protocol. In contrary, the study in [12] has underestimated the discontinuity protocol used in LTE for the energy efficiency by arguing that the method may not necessarily be vital for one end to other.

\section{K. A. Dotche}

College of Technology / University of Education Winneba Kumasi-Ghana
The coverage ratio to the Base Transceiver station (BTS) optimal transmission has been investigated in [13]. In [14], a cooperative communication (CC) in a cognitive radio network (CRN) was considered and the network coding was used to increase the system capacity. In [15], the work has introduced a traffic-aware mechanism, named cooperative communication [16] of BS sleep mode for an effective radio resource management [17] as a case in the distributed cellular networks [18]. In [19], the results have shown that deploying more BSs, the throughput may be increased significantly. In [20], Pareto optimal set point was considered in improving both the spectrum and energy efficiency. Chunlong et al in [21], have investigated the energy efficiency maximization in multitransmitters' downlink networks. They have proposed a novel algorithm for power allocation while solving the singleobjective optimization problem.

This paper investigates an optimization of the spectrum and energy efficiency using coverage sensitivity criterion, under the absence of user's activities. This constraint aims at deleting empty transmission block where no cell load is detected.

The paper is structured as follows. The section II depicts the system modelling to some identified constraints. The section III presents the simulation parameters and the software used. Section IV discusses the obtained results. The conclusion is given in the section $\mathrm{V}$.

\section{Theoretical System Modelling}

A tier heterogeneous network is assumed.

\section{A. Problem Description}

A multi-transmitters' OFDMA downlink system with a flat fading channel. The femto-cells, and pico-cells, relays and the central base station (CBS, eNodeB) are connected to the core network through fiber backhaul. The network is a tier heterogeneous network consisting of type-I relays (nontransparent relays, this special case to be viewed as a cell for the modelling purpose), femto-cells, and pico-cells. The transmitters could be viewed as remote antenna unit (RAI). Assuming that users are served by the nearest transmitter (RAI, cell) and the channel state information (CSI) is known by the BSs. Consequently, some of the cells may be inactive. Inactive cell is a cell that is not servicing any user. 


\section{B. Optimization of the Energy and Spectrum Efficiency}

Assuming that there are $\mathrm{M}$ users (MSs) and $\mathrm{N}$ transmitters in a given coverage area of the multi-transmitters system. The relays, femto, pico and macro-cell antenna are independent transmitters. The transmitters may be viewed as remote antenna units. The macrocell antenna is a special case of the transmitters (to which we assign a function of the central base station CBS).

Recalling, if a user, $S_{i}$ connected to the active node $n$, it comes that the probability for a cell to be active $p_{a_{n, i}}$ is given as [19]:

$$
p_{a_{n, i}}=1-(1+1 /(\mu \cdot \rho))^{-\mu}
$$

with the BS density, $\lambda_{b}$-user density, $\lambda_{u}$ the BS-User density ratio $\rho \triangleq \frac{\lambda_{b}}{\lambda_{u}}$ and $\mu$ is a constant related to the cell-size distribution. The signal to noise ratio (SNR), $\gamma_{i}$, at $M S_{i}$ is given as in [20]:

$$
\gamma_{i}=\frac{\sum_{n=1}^{N} p_{n, i} \cdot\left|h_{n, i}\right|^{2}}{\sigma_{z}^{2}}
$$

with $p_{n, i}$ transmit power of $M S_{i}$, and $h_{n, i}$ fading channel experienced by $M S_{i}$, and $\sigma_{z}^{2}$ is the Additive White Gaussian Noise (AWGN). When, assuming an M-QAM coherent modulation to achieve Shannon data rate limit, the spectral efficiency of $M S_{n, i}$ is expressed as in (3)

$$
\eta_{S E}(i)=\log _{2}\left(1+\beta \gamma_{i}\left(p_{n, i}\right)\right)
$$

The BER, $p_{e}$, for coherently detected M-QAM with Gray mapping over an AWGN channel is related to the $\mathrm{SNR}, \gamma_{i}$, as given in [21].

where

$$
p_{e}=0.2 \exp \left(-\frac{1.5 \gamma_{i}}{M-1}\right)
$$

$$
\beta=\frac{(M-1)}{\gamma_{i}}=-\frac{1.5}{\ln 5 p_{e}}
$$

The EE, $\gamma_{E E}(A)$ in a given area is defined as the total spectral efficiency (TSE) $\eta_{A}$ of users within the cell area A over the total transmit power $P_{t}(A)$ and accounting for the circuit power and non-transmission power consumption of inactive cells (idle transmitters) $p_{c}(A)$. Then, $\gamma_{E E}(A)$, is given as in (4)

with

$$
\gamma_{E E}(A)=\frac{\eta_{A}}{P_{t}(A)+p_{c}(A)}
$$

$$
\eta_{A}=\sum_{i=1}^{M} \eta_{S E}(i)
$$

a linear power consumption is assumed at a connected node, $P_{T, i}$, the standard formulation is as follows:

$$
P_{T, i}=\frac{1}{\eta} P_{t, i}+\alpha P_{c}+P_{0}
$$

$\eta$ is the efficiency of power amplifier, $p_{t, i}$, the transmit power of the node, $\alpha$ the number of antennas at the node, the circuit power $P_{c}$, and $P_{0}$ is the non-transmission power consumption including baseband signal processing, battery backup, cooling etc. The transmit power $P_{a_{n, i}}$ of an active node, could be written as in (6):

$$
P_{a_{n, i}}=\frac{\lambda_{b} \cdot p_{a_{n, i}} \cdot P_{t}}{\eta}
$$

Therefore, the quantities in (4) are as follows:

$P_{t}(A)=\sum_{i=1}^{M} \sum_{n=1}^{N} P_{a_{n, i}}$

$p_{c}(A)=\lambda_{b}\left(P_{0}+p_{a_{n, i}} . P_{c}\right)$, is related to the total nontransmission power consumption $\left(P_{0}\right)$ from inactive nodes and the circuit power $\left(P_{c}\right)$.

\section{Problem Formulation}

The SE optimization in multi-transmitters' system is as in (7) $\operatorname{Max} \eta_{A}$

Subject to

$$
\begin{gathered}
P_{a_{n, i}} \in\left[0, P_{n}^{\max }\right] \\
\sum_{i=1}^{M} P_{a_{n, i}} \leq P_{n}^{\max }
\end{gathered}
$$

where $P_{n}^{\max }$ denotes the maximum transmit power of the transmitter $\mathrm{n}$, and the modulation margin optimization is as in (8):

$$
\operatorname{Min} \sum_{i=1}^{M} \sum_{n=1}^{N} P_{a_{n, i}}
$$

Subject to $P_{a_{n, i}} \in\left[0, P_{n}^{\max }\right]$

$$
\log _{2}\left(1+\beta \gamma_{i}\left(P_{a_{n, i}}\right)\right) \geq \eta_{S E}(i)
$$

The optimization of the EE is formulated as in (9):

$$
\operatorname{Max} \gamma_{E E}(A)
$$

Subject to

$$
\begin{aligned}
& P_{a_{n, i}} \in\left[0, P_{n}^{\max }\right] \\
& \sum_{i=1}^{M} P_{a_{n, i}} \leq P_{n}^{\max }
\end{aligned}
$$

This yields a multi objective problem as in (10):

$$
\begin{gathered}
\operatorname{Min} \sum_{i=1}^{M} \sum_{n=1}^{N} P_{a_{n, i}} \\
\operatorname{Max} \sum_{i=1}^{M} \log _{2}\left(1+\beta \gamma_{i}\left(P_{a_{n, i}}\right)\right)
\end{gathered}
$$

Subject to

$$
\begin{gathered}
P_{a_{n, i}} \in\left[0, P_{n}^{\max }\right] \\
\sum_{i=1}^{M} P_{a_{n, i}} \leq P_{n}^{\max } \\
\log _{2}\left(1+\beta \gamma_{i}\left(P_{a_{n, i}}\right)\right) \geq \eta_{S E}(i) \\
\lambda_{b}\left(P_{0}+p_{a_{n, i}} P_{c}\right) \leq p_{c} \\
C_{n, i} \leq p_{n, i}-P_{a_{n, i}} \leq 0
\end{gathered}
$$


Proc. of the Fourth International Conference on Advances in Computing, Electronics and Communication - ACEC 2016. Copyright ( $)$ Institute of Research Engineers and Doctors. All rights reserved.

ISBN: 978-1-63248-113-9 doi: 10.15224/ 978-1-63248-113-9-06

(14) formulates the constraint of the circuit power and nontransmission power consumption threshold, and (15) is related to the coverage sensitivity $C_{n, i}$ defined in [22] of the $M S_{i}$, with $p_{n, i}$ the transmit power of the mobile station to the node $\mathrm{n}$ . Thus, the goal is to minimize the transmission power not only but also to maximize the SE. The coverage sensitivity (Cr) null may fulfill the condition of the absence of user activity.

\section{Methodology and Simulation}

MatLab software is used for the simulation and to solve the multi-objective problem using the fminimax multi-objective function. The following parameters were considered as in Table 1.

\section{TABLE 1: Simulation PARAMETERS}

\begin{tabular}{|l|l|c|c|}
\hline \multirow{2}{*}{$\begin{array}{l}\text { Table } \\
\text { Head }\end{array}$} & \multicolumn{3}{|c|}{ Simulation Parameters } \\
\cline { 2 - 4 } & Description & Symbol & Value \\
\hline & M- QAM & $\mathbf{6 4 ; 2 5 6}$ \\
\hline & Number of Users & 50 \\
\hline & the circuit power & Ptx(CBS) & $40 \mathrm{dBw}$ \\
\hline & $\begin{array}{l}\text { the non-transmission power } \\
\text { consumption }\end{array}$ & $P_{c}$ & 100 \\
\hline & $\begin{array}{l}\text { the transmit power of an active } \\
\text { node }\end{array}$ & $p_{a_{n, i}}$ & $5 \mathrm{dBm}$ \\
\hline & $\begin{array}{l}\text { Number of active nodes } \\
\text { N }\end{array}$ & 5 \\
\hline & $\begin{array}{l}\text { constant related to the cell-size } \\
\text { distribution }\end{array}$ & $\mathrm{a}$ & 0.6 \\
\hline & $\begin{array}{l}\text { User density } \\
\text { BS density }\end{array}$ & $\mu=\mathrm{u}$ & 0.95 \\
\hline & $\begin{array}{l}\text { BS-User density } \\
\lambda_{b}=\mathrm{b}\end{array}$ & 0.33 \\
\hline & $\begin{array}{l}\text { variance of AWGN channel } \\
\mathrm{d}=\mathrm{b} / \mathrm{u}\end{array}$ \\
\hline & environment fading & $\mathrm{D}$ & 25 \\
\hline
\end{tabular}

\section{Results and Discussion}

In the Figures 1-9 are showing some preliminary results with respect to the coverage sensitivity criterion $(\mathrm{Cr})$.
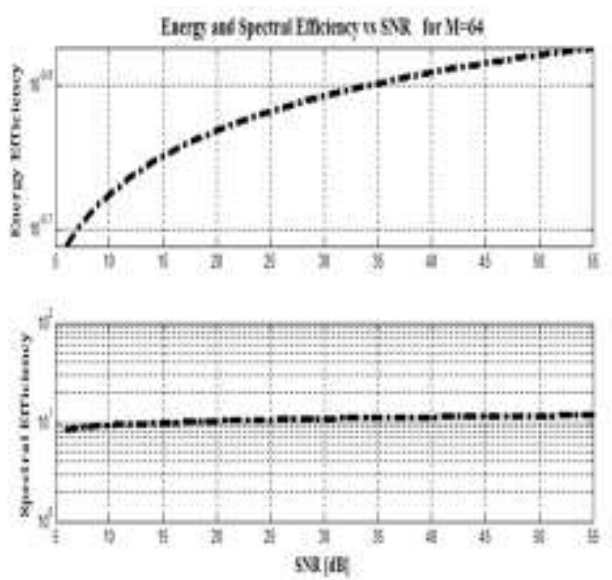

Fig. 1
In Fig. 1, the results are depicting the spectral efficiency (SE) and the energy efficiency (EE) in some subplots' comparison against the signal to noise ratio (SNR). In Fig. 2, the coverage sensitivity criterion $(\mathrm{Cr}[\mathrm{dB}])$ is considered. The comparative results in Fig. 2 against the SNR, and that of the $\mathrm{Cr}$ in Fig. 3 may indicate that when using the latter, a faster decrease in the power consumption 1 may be achieved though the SE indicates a slow increased curve. For instance, in Fig.2, for $M=64$, the EE approaches $\mathbf{1 0}^{-\mathbf{0 . 4}}$ and the SE, which is about $10^{2}$.
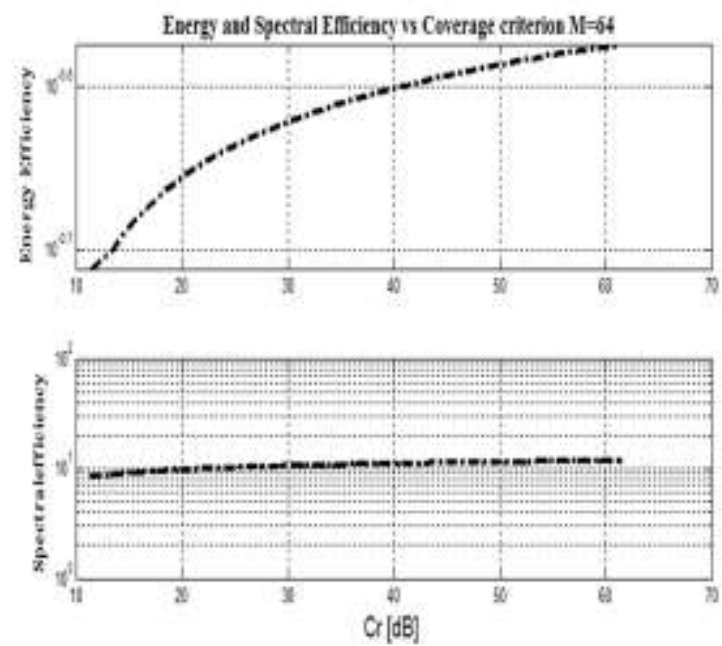

Fig. 2
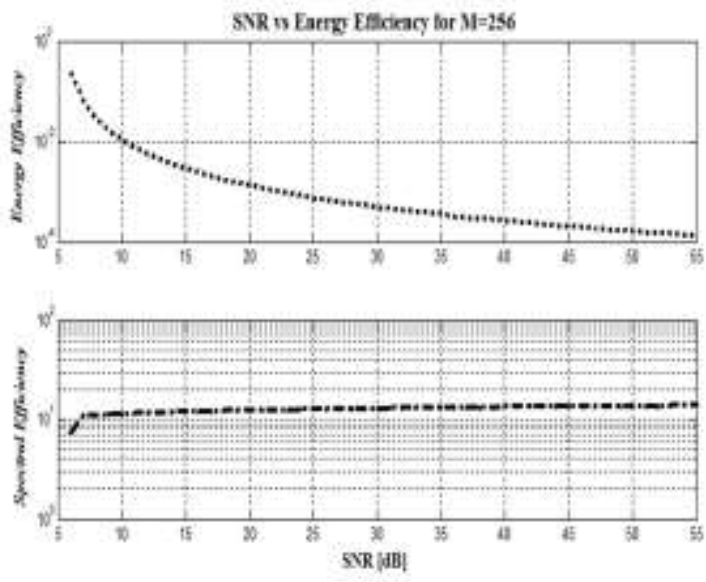

Fig. 3

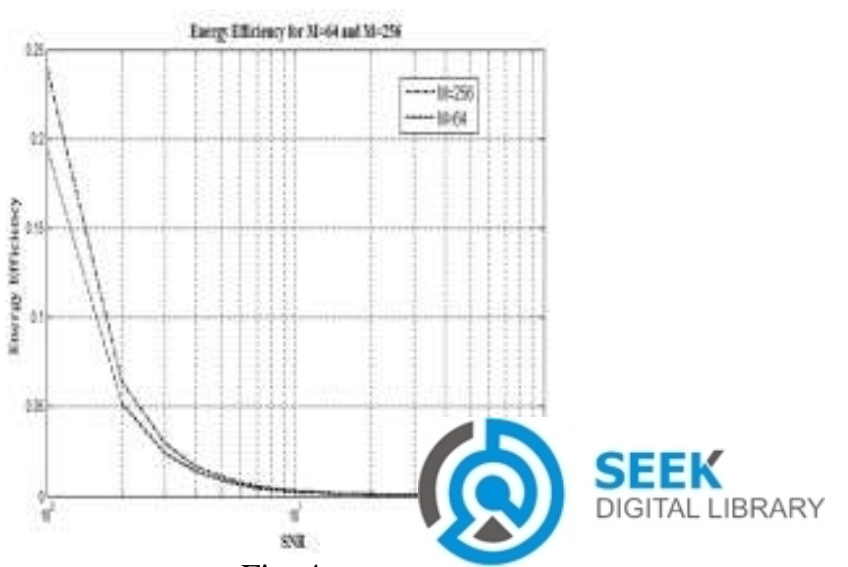

Fig. 4 
Proc. of the Fourth International Conference on Advances in Computing, Electronics and Communication - ACEC 2016.

Copyright ( $)$ Institute of Research Engineers and Doctors. All rights reserved.

ISBN: 978-1-63248-113-9 doi: 10.15224/ 978-1-63248-113-9-06

In Fig. 3, a high order modulation is considered $(\mathrm{M}=256)$, with emphasis on the SE maximization, and EE minimization.

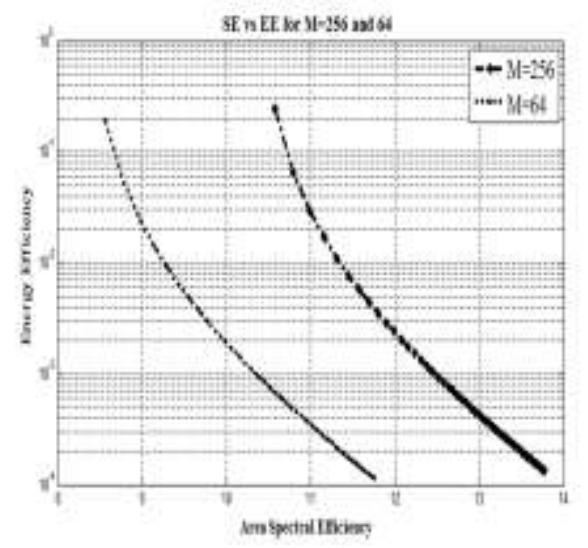

Fig. 5

It shows that the energy consumption is markedly reduced
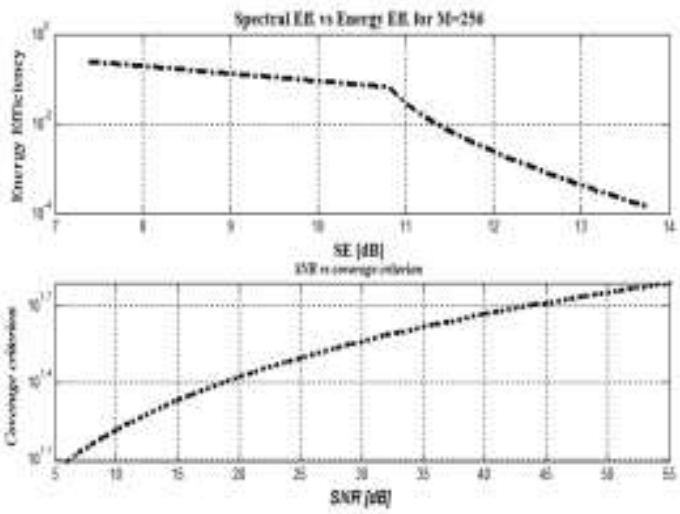

Fig. 6

$\left(\mathrm{M}=256, \mathrm{EE} \sim \mathbf{1 0}^{-\mathbf{0 . 8}}\right.$ There will be a small amount of energy consumed to keep the transmitters up, when they are in idle mode; this amount of energy may be negligible. This may be

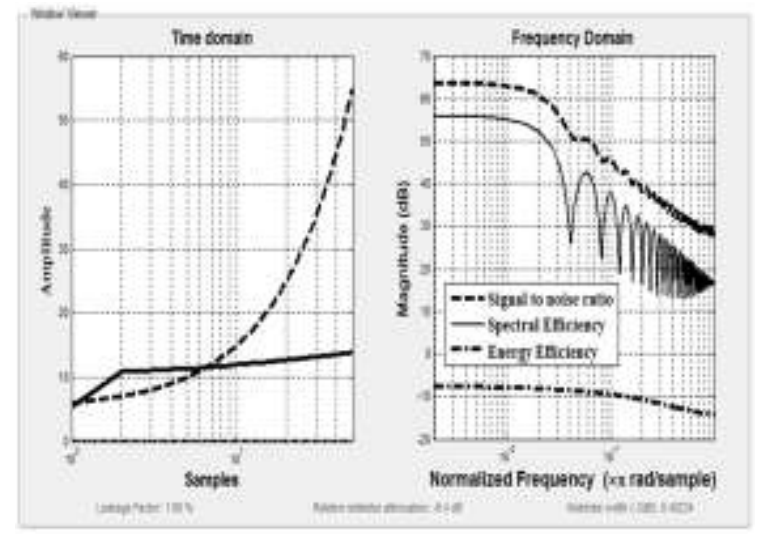

an

Fig. 7

agreement with the asymptotic decrease in the EE when one assumes an infinite size of transmission block. A higher order modulation scheme may make more power demand at expense of a decrease in the SE (see Fig. 5) as in [5]. Though in Fig. 5, it may indicate that both graph will cross each other. This may be explained by the fact that the energy consumption is decreasing but the SE keeps on increasing. For $M=256$, the EE is high at the beginning and $\mathrm{d} 10^{-0.51}$. The SE for $(\mathrm{M}=256)$ is about $10^{1.13}$, this is lower than that of the observed results in Fig. 1 and 2.

In Fig. 6, it is depicting the EE against the SE. A break down point is obtained at a higher modulation $(M=256)$ as in [14]. It is noting that though the SE increases, the energy consumption is reduced. The second subplot in Fig. 6 presents a ratio of the $\mathrm{Cr}$ to the SNR. It may be argued that the assumption of the absence user's activity may be assumed in nature to a few

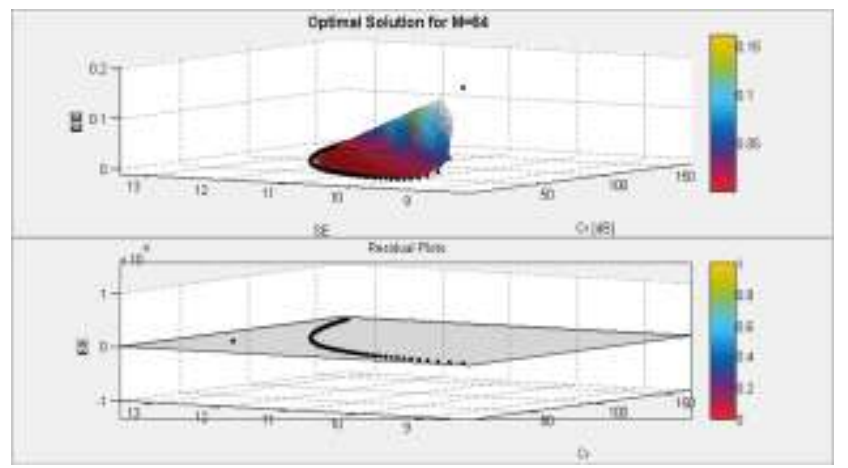

Fig. 8

users using a larger available bandwidth and may result in a systematic decrease in EE. The SE, EE and the SNR are given in frequency and time domain in Fig. 7. The power

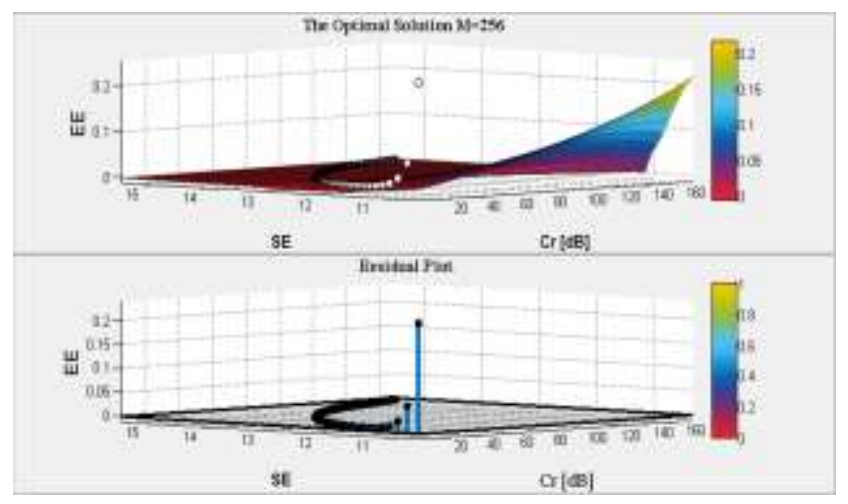

Fig. 9

consumption is significantly reduced. The optimal solutions are given in Fig. 8 and 9 respectively for $M=64$ and $M=256$. The $\mathrm{Cr}$ was also used in the fitting tool in order to get accurate optimal solutions in a 3D plot. The goodness of fit parameters for $\mathrm{M}=256$ are as follows: SSE: 0.0007108, R-square: 0.9881, Adjusted R-square: 0.9855 and RMSE: 0.004199, meanwhile for the $\mathrm{M}=64$, SSE: $2.121 \mathrm{e}-34$ and R-square: 1 . 
Proc. of the Fourth International Conference on Advances in Computing, Electronics and Communication - ACEC 2016. Copyright (C) Institute of Research Engineers and Doctors. All rights reserved.

ISBN: 978-1-63248-113-9 doi: 10.15224/ 978-1-63248-113-9-06

\section{Conclusion}

The paper investigated an optimization of both the spectrum and energy efficiency by formulating and solving a multiobjective problem under a novel constraint of coverage sensitivity criterion $(\mathrm{Cr})$. The work uses the Matlab simulation and fminimax function to solve the multi-objective problem, in addition to an enhanced fitting curve to obtain the optimal solutions given in Fig. 8 and 9. For a high order modulation scheme (256-QAM) show high spectral area (Fig. 9), while for a low order this is a slow increase curve. The obtained results may indicate that when using the $\mathrm{Cr}$ the $\mathrm{EE}$ is significantly reduced. The transmitters' power consumption is reduced, and the energy consumed to keep the transmitters up, in idle mode is significantly negligible. This contribution remains in line of exploring efficient strategies for SE and EE in cooperative communication. In future, this criterion will be embedded onto a chip for a network sensing capability.

\section{References}

[1] J. D. Parsons, The Mobile Radio Propagation Channel, London: John Wiley and Sons, Second Edition, 1992.

[2] M. Rahnema, UMTS Network planning, optimization and interoperation with, John Wiley \& Sons, Inc, 2008.

[3] R. M. Ajay, Fundamentals of Cellular Network Planning and Optimization, John Wiley and Sons, 2004.

[4] C. Y. Samuel, CDMA RF Engineering, ARTECH HOUSE, INC, 1998.

[5] I. Seo, S. Won and Y. Kim, "Performance Analysis of High Order Modulation Schemes in the wireless Communication System," Gangwon-Do, 2007.

[6] W. Zhuang and Y. Zhou, "A Survey of Cooperative MAC Protocols for Mobile Communication Networks," 2013.

[7] Luis M. et al., "Challenges and Enabling Technologies for Energy Aware in Mobile Radio Network," IEEE Communications Magazine, pp. 66-72, 2010.

[8] Daquan Feng et al, "A Survey of Energy-Efficient Wireless
Communications," IEEE COMMUNICATIONS SURVEYS \& TUTORIALS, VOL. 15, NO. 1, pp. 162-178, 2013.

[9] Ziaul Hasan et al, "Green Cellular Networks: A Survey, Some Research Issues and Challenges," International Journal of Emerging Technology and Sciences, 2013.

[10] Guowang Miao et al., "Cross-Layer Optimization for EnergyEfficient Wireless Communications: A survey," 2013.

[11] B. Oliver, Z. Dietrich and B. Ulrich, "Approaches to Energy Efficient Wireless Access Networks".

[12] Andrews. J, " Interference cancellation for cellular systems: A contemporary Overview," IEEE Wirel Commun., Vols. 12, 1929 MWC.2005.1421925, no. 2, 2005.

[13] Zhou, Liu, Pan, Tian, Shi, and Yang, "Two-Stage Cooperative Multicast Transmission with Optimized Power Consumption and Guaranteed Coverage," 2013.

[14] Li, Guo, Zhuang and Ye, "On Efficient Resource Allocation for Cognitive and Cooperative Communication," 2013.

[15] Han, Yang and Molisch, "Spectrum and Energy Efficient Cooperative Base Station Doze," 2013.

[16] a. Z. Xu, "Throughput Optimal Policies for Energy Harvesting Wireless Transmitters with non Ideal Circuit Power," 2013.

[17] Holtkamp et al., "Minimizing Base Station Power Consumption," 2013.

[18] Lopez-Perez et al., "Power Minimization Based Resource Allocation for OFDMA FemtoCell Networks," 2013.

[19] C. Li, J. Zhang and K. B. Letaief, "Energy Efficiency Analysis in Small-Cell Networks," vol. 1306.6169v1, 2012.

[20] Zhihang et al., "Energy spectral efficiency tradeoff in downlink OFDMA network," 2014.

[21] Chunlong et al., "Energy Efficiency and Spectral Efficiency Tradeoff in Downlink Distributed Antennas systems," vol. 1, no. 3, 2012, June.

[22] K. Blitti, K. A. Dotche and K. Diawuo, "Macrocell Antenna Downtilt to Field Data Study in Ghana," in IEEE-ICAST, Kumasi, 2012.

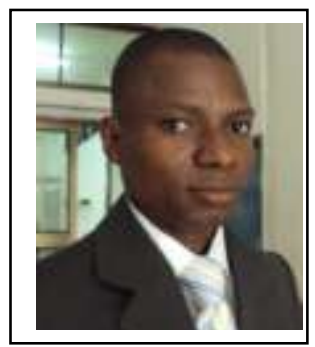

K. A. Dotche

K. A. Dotche is a holder of an MSc. Telecom. Eng. from the College of Engineering (KNUST) in 2010. He is currently a lecturer with the Department Electrical Technology at the University of Education Winneba, Kumasi-Ghana . He also teaches at Ecole Nationale Supérieure d'Ingénieurs (ENSI), University of Lomé in Togo. His research interests comprise Energy efficiency in wireless sensor networks, antennas and E-M propagation in

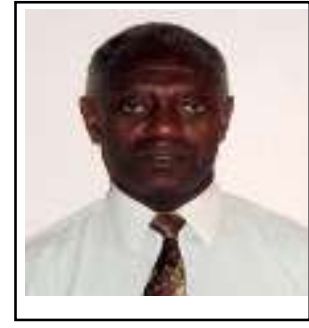

Willie K. Ofosu

Willie K. Ofosu is a Professor of Engineering at Penn State Wilkes-Barre. He is a member of ASEE, IEEE, IET, and a Chartered Engineer (CEng) of England. His research interests include various aspects of Telecommunications, Solar Technology, globalization and technology transfer. He is involved in teaching and research activities in Ghana, West Africa. He received his $\mathrm{Ph} . \mathrm{D}$. from the Electronic Systems Engineering Department at University of Essex in England. 